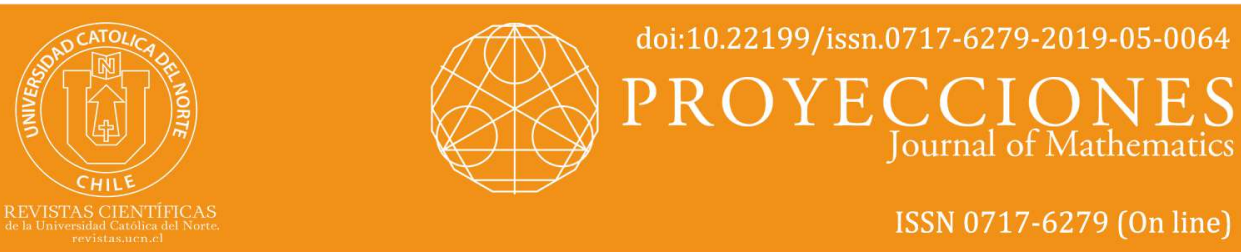

\title{
Zero forcing in Benzenoid network
}

J. Anitha*

Indra Rajasingh ${ }^{* *}$

*Easwari Engineering College, Dept. of Mathematics, Chennai, TN, India.

$\nabla$ anithaharish78@gmail.com

${ }^{* *}$ Vellore Institute of Technology, School of Advanced Sciences, Chennai, TN, India.

$\mathbf{v}$ indra.rajasingh@vit.ac.in

Received: September 2018 | Accepted: January 2019

\section{Abstract:}

$A$ set $S$ of vertices in a graph $G$ is called a dominating set of $G$ if every vertex in $V(G) \backslash S$ is adjacent to some vertex in $S$. A set $S$ is said to be a power dominating set of $G$ if every vertex in the system is monitored by the set $S$ following a set of rules for power system monitoring. The power domination number of $G$ is the minimum cardinality of a power dominating set of $G$. A dynamic coloring of the vertices of a graph $G$ starts with an initial subset $S$ of colored vertices, with all remaining vertices being noncolored. At each discrete time interval, a colored vertex with exactly one non-colored neighbor forces this non-colored neighbor to be colored. The initial set $S$ is called a forcing set (zero forcing set) of $G$ if, by iteratively applying the forcing process, every vertex in $G$ becomes colored. The zero forcing number of $G$, denoted $Z(G)$, is the minimum cardinality of a zero forcing set of $G$. In this paper, we obtain the zero forcing number for certain benzenoid networks.

Keywords: Zero forcing set; Pyrene networks; Circum-pyrene networks; Circum-trizene networks.

MSC (2010): 05C69, 05C85, 05C90, 05C20.

\section{Cite this article as (IEEE citation style):}

J. Anitha and I. Rajasingh, "Zero forcing in Benzenoid network", Proyecciones (Antofagasta, On line), vol. 38, no. 5, pp. 999-1010, Dec. 2019, doi: 10.22199/issn.0717-6279-2019-05-0061. [Accessed dd-mm-yyyy].

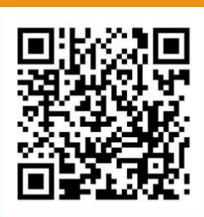

Article copyright: (c) 2019 J. Anitha and Indra Rajasingh. This is an open access article distributed under the terms of the Creative Commons Licence, which permits unrestricted use and distribution provided the original author and source are credited. 


\section{Introduction}

The idea of zero forcing on graphs is a current thought that is part of a program study on minimum ranks of matrices with particular combinatorial limitations (American Institute of Mathematics 2008). Zero forcing is utilized to study inverse eigenvalue problems, PMU placement problems, and quantum control problems [1]. Zero forcing is also called graph infection or graph propagation in the zones identified with quantum dynamics and control theory of quantum mechanical systems [2]. By the monotonous utilization of a similar quantum transformation, this reality has been used to accomplish noise protection, cooling, state preparation, quantum state transfer and in computer science in the context of fast-mixed searching [2].

Recently, there has been a lot of interest in studying the forcing number of graphs for its own sake, and its relation to other graph parameters, such as the path cover number, connected domination number, and the chromatic number. Among others, zero forcing number of a graph contain upper bounds in terms of its degrees [3]. It is easy to see that the trivial lower bound on the zero forcing number of a graph is $Z(G) \geq \delta-1$.

A benzenoid system, the so called benzenoid graph or hexagonal system, is a finite connected planar graph with no cut vertices in which every interior region is bounded by a regular hexagon. Polycyclic hydrocarbons formed from $n$ condensed benzenoid rings are the most important carcino-genics: they are classified into two classes catacondensed and peri-condensed. By means of dualist graph (consisting from the points corresponding to centres of hexagons, connected by lines whenever two hexagons are condensed). Dualist graph of catacondensed are trees and of peri-condensed contains cycle. Peri-condensed benzenoid graphs pose significant challenges due to their very nature of complex connectivity, as their dualists are not trees. Peri-condensed compounds, their circum-scribings and circumcisions find novel applications ranging from synthesis of new molecules such as Kekulenes [7].

Definition 1.1. [4] For $v \in V(G)$, the open neighbourhood of $v$, denoted as $N_{G}(v)$, is the set of vertices adjacent with $v$; and the closed neighbourhood of $v$, denoted by $N_{G}[v]$, is $N_{G}(v) \cup\{v\}$. For a set $S \subseteq V(G)$, the open neighbourhood of $S$ is defined as $N_{G}(S)=\bigcup_{v \in S} N_{G}(v)$ and the closed neighbourhood of $S$ is defined as $N_{G}[S]=N_{G}(S) \cup S$. For brevity, we denote $N_{G}(S)$ by $N(S)$ and $N_{G}[S]$ by $N[S]$. 
Definition 1.2. [4] For a graph $G(V, E), S \subseteq V$ is a dominating set of $G$ if every vertex in $V \backslash S$ has at least one neighbour in $S$. The domination number of $G$, denoted by $\gamma(G)$, is the minimum cardinality of a dominating set of $G$.

Definition 1.3. [5] Let $G(V, E)$ be a graph and let $S \subseteq V(G)$. We define the sets $M^{i}(S)$ of vertices monitored by $S$ at level $i, i \geq 0$, inductively as follows:

1. $M^{0}(S)=N[S]$.

2. $M^{i+1}(S)=M^{i}(S) \cup\left\{w: \exists v \in M^{i}(S), N(v) \cap\left(V(G) \backslash M^{i}(S)\right)=w\right\}$.

If $M^{\infty}(S)=V(G)$, then the set $S$ is called a power dominating set of $G$. The minimum cardinality of a power dominating set in $G$ is called the power domination number of $G$ written $\gamma_{p}(G)$.

Definition 1.4. [5] The zero forcing process is a coloring game on a graph. If $u$ is a blue vertex and exactly one neighbor $w$ of $u$ is white, then change the color of $w$ to blue; this is called the color change rule and we say that $u$ forces $w$. A zero forcing set for $G$ is a subset of vertices $B$ such that if initially the vertices in $B$ are colored and the remaining vertices are colored white, then repeated application of the color change rule can color all vertices of $G$ blue. A minimum zero forcing set is a zero forcing set of minimum cardinality, and the zero forcing number $Z(G)$ of $G$ is the cardinality of a minimum zero forcing set.

\section{Main Result}

In this section, we solve the zero forcing problem for certain benzenoid networks.

In 2015 Ferrero et al. [5] proved the following theorem which shows the relationship between zero forcing set and power dominating set.

Theorem 2.1. [5] Let $G$ be a graph. Then $\left\lceil\frac{Z(G)}{\Delta(G)}\right\rceil \leq \gamma_{p}(G)$ and this bound is tight.

In 2017 we obtained a sharp lower bound for certain class of benzenoid networks described in Theorem 2.1 with respect to power domination number [6]. In this paper, we solve the zero forcing problem for such classes of benzenoid networks.

The following lemma establishes a critical subgraph $H$ of $G$ in the sense that $H$ contains at least one vertex of any zero forcing set. 
Lemma 2.2. Let $G$ be a graph and $H$ as shown in Figure 1 be a subgraph $G$ with $\operatorname{deg}_{H} w_{i}=\operatorname{deg}_{G} w_{i}=2, \forall i, i=1,2,3,4$. Then $H$ is a critical subgraph of $G$.

Proof. Neither $u$ nor $v$, when monitored, can further monitor any of $w_{i}, i=1,2,3,4$, as $\operatorname{deg}_{H} u=\operatorname{deg}_{H} v=3$.

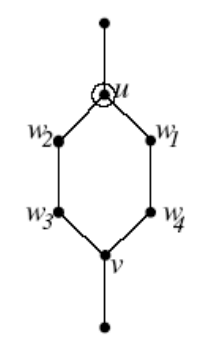

Figure 1: Circled vertices constitute a zero forcing set of $G$

\subsection{Zero Forcing of Pyrene Network}

Pyrene is an alternante polycyclic aromatic hydrocarbon $(P A H)$ and consists of four fused benzene rings, resulting in a large flat aromatic system. It is a colorless or pale yellow solid which forms during incomplete combustion of organic materials and therefore can be isolated from coal tar together with a broad range of related compounds. In the last four decades, a number of research works have been reported on both the theoretical and experimental investigation of pyrene concerning its electronic structure, $U V$ vis absorption and fluorescence emission spectrum. Indeed, this polycyclic aromatic hydrocarbon exhibits a set of many interesting electrochemical and photophysical attributes, which have result in its utilization in a variety of scientific areas. Like most PAHs, pyrene is used to make dyes, plastics and pesticides. Figure 2(b) depicts the graph of Circum-pyrene (1). Inductively, circum-pyrenes $(n)$ is obtained from circum-pyrenes $(n-1)$ by adding a layer of hexagons around the boundary of circum-pyrenes $(n-1)$. Similar construction follows for circum-trizenes $(n)$. See Figure 2(c) [7].

Lemma 2.3. Let $G$ be a pyrene network $P Y(n), n \geq 2$. Then $Z(G) \geq n$.

Proof. In $P Y(n)$, there are $n$ critical subgraphs, each isomorphic to $H$ as described in Lemma 2.2. Therefore, $Z(G) \geq n$. 


\section{Zero Forcing Algorithm in Pyrene Network}

Input: Pyrene network $P Y(n), n \geq 2$.

Algorithm: Name the vertices of $P Y(n), n \geq 2$ as 1 to $2 n^{2}+4 n$ sequentially from left to right, row wise beginning with the top most row. Select the vertices $\{2,4\} \cup\{6,12,20, \ldots n \times(n+1)\}$ in $S_{n}$. See Figure $2(a)$.

Output: $Z(P Y(n))=n+1$.

Proof of Correctness: Every vertex in $S_{n}$ is adjacent to exactly one noncolored vertex which in turn forces exactly one non-colored vertex. Then $S_{n}$ is a zero for cing set of $P Y(n)$. This implice that, $Z(P Y(n))=n+1$. Hence the proof.

Theorem 2.4. Let $G$ be a pyrene network $P Y(n), n \geq 2$. Then $Z(G)=$ $n+1$.

Lemma 2.5. Let $G$ be a circum-pyrene $(n), n \geq 1$. Then $Z(G) \geq 2 n+2$.

Proof. In circum-pyrene (n), there are $2 n+2$ critical subgraphs, each isomorphic to $\mathrm{H}$ as described in Lemma 2.2. Therefore, $Z(G) \geq 2 n+2$.

\section{Zero Forcing Algorithm in Circum-Pyrenes $(n)$}

Input: Circum-pyrenes $(n), n \geq 1$.

Algorithm: Name the vertices of Circum-pyrenes $(n), n \geq 1$ as 1 to $6 n^{2}+20 n+16$, sequentially from left to right, row wise beginning with the first row. Consider $2 n+2$ hexagons in the outer most layer of the Circumpyrenes $(n)$. Let $P^{*}$ denote the path induced by the edges of the hexagons that are not boundary edges of any other hexagon. Select $2 n+2$ vertices of degree 2 in $P^{*}$, which are at distance 2 apart on $P^{*}$. Apart from these vertices, select one more vertex in $S_{n}$ from $P^{*}$, adjacent to any one vertex which is selected already. See Figure 2(b).

Output: $Z($ Circum-pyrenes $(\mathrm{n}))=2 n+3$. 
Proof of Correctness: $S_{n}$ is a zero forcing set of Circum-pyrene $(n)$ with $\left|S_{n}\right|=2 n+3$. See Figure $2(b)$. Every vertex in $S_{n}$ is adjacent to exactly one-non colored vertex. Now $S_{n}$ is a zero forcing set of Circum-pyrene $(n)$. This implies that, $Z($ Circum - pyrene $(\mathrm{n}))=2 n+3$. Hence the proof.
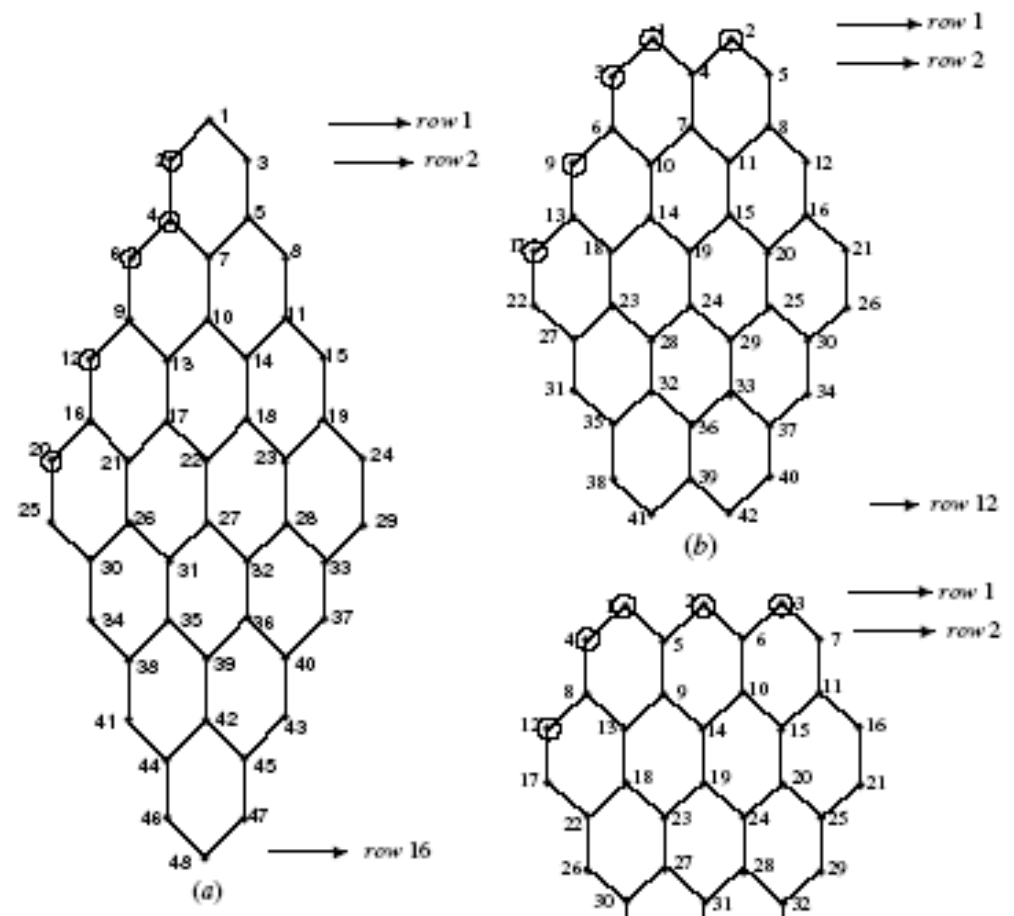

(b)

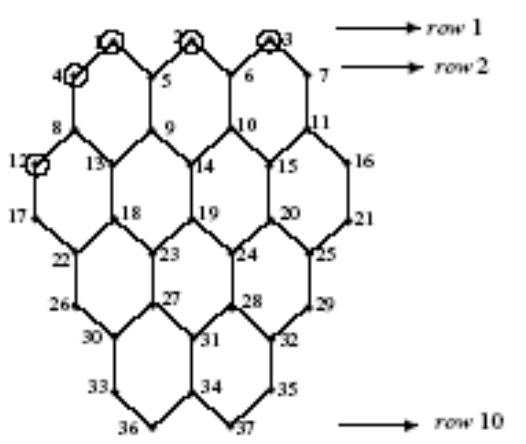

(c)

Figure 2: Circled vertices constitute (a) a minimum zero forcing set of pyrene $P Y$ (4) (b) a minimum zero forcing set of circum-pyrene(1) (c) a minimum zero forcing set of circumtrizenes(1)

Lemma 2.6. Let $G$ be a circum-trizenes $(n), n \geq 1$. Then $Z(G) \geq 2 n+2$. 
Proof. In circum-trizenes $(n)$, there are $2 n+2$ critical subgraphs, each isomorphic to $\mathrm{H}$ as described in Lemma 2.2. Therefore, $Z(G) \geq 2 n+2$.

\section{Zero Forcing Algorithm in Circum-Trizenes}

Input: Circum-trizenes $(n), n \geq 1$.

Algorithm: Name the vertices of $\operatorname{Circum-trizenes}(n), n \geq 1$ as 1 to $6 n^{2}+18 n+13$ sequentially from left to right, row wise begining with the first row. Consider $2 n+2$ hexagons in outer most layer of the Circumpyrenes $(n)$. Let $P^{*}$ denote the path induced by the edges of the hexagons that are not boundary edges of any other hexagon. Select $2 n+2$ vertices of degree 2 in $P^{*}$, which are at distance 2 apart on $P^{*}$. Apart from these vertices, select one more vertex in $S$ from $P^{*}$, select one more vertex in $S_{n}$ from $P^{*}$, adjacent to any one vertex which is selected already. See Figure $2(c)$.

Output: $Z($ Circum-trizenes $(\mathrm{n}))=2 n+3$.

Proof of Correctness: Every vertex $v \in S_{n}$ is adjacent to exactly onenon colored vertex. Proceeding inductively, every vertex in the graph is colored. Now $S_{n}=2 n+3$. This implies that, Circum-pyrene $(n)=2 n+3$. Hence the proof.

Theorem 2.7. Let $G$ be a circum-pyrenes $(n)$ or a circum-trizenes $(n)$, $n \geq 1$. Then $Z(G)=2 n+3$.

\subsection{Zero Forcing of Circum-Coronene}

Coronene is generated by circumscribing the benzene $\left(\mathrm{C}_{6} \mathrm{H}_{6}\right)$ hexagon with a rim of 6 hexagons $(\mathrm{C} 18 \mathrm{H} 6)$. Since benzene is the only possible $\left(\mathrm{C}_{6} \mathrm{H}_{6}\right)$ hexagonal isomer, coronene is the only possible $\left(\mathrm{C}_{24} \mathrm{H}_{12}\right)$ isomer composed of strictly hexagonal rings. Benzene is the excised internal structure of coronene. Circumscribing coronene $\left(\mathrm{C}_{24} \mathrm{H}_{12}\right)$ gives circum-coronene $\left(\mathrm{C}_{54} \mathrm{H}_{18}\right)$.

Lemma 2.8. Let $G$ be a circum-coronene $(n), n \geq 1$. Then $Z(G) \geq 2 n+3$. 
Proof. In circum-coronene $(n)$, there are $2 n+3$ critical subgraphs, each isomorphic to $\mathrm{H}$ as described in Lemma 2.2. Therefore, $Z(G) \geq 2 n+3$.

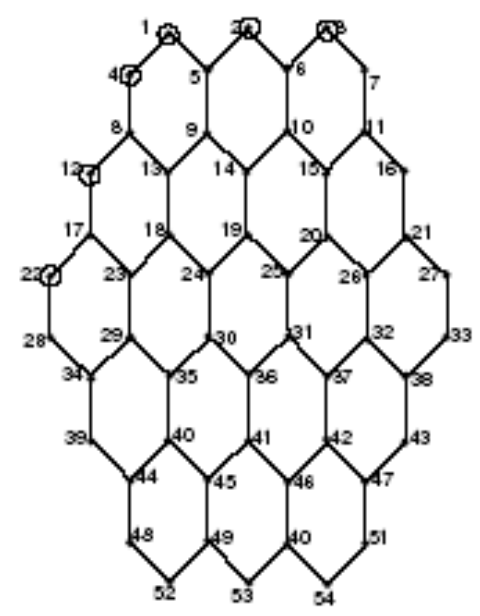

(a)

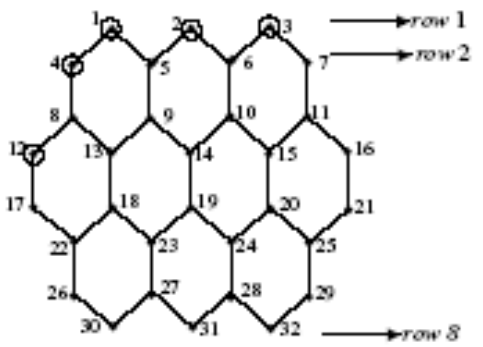

(b)

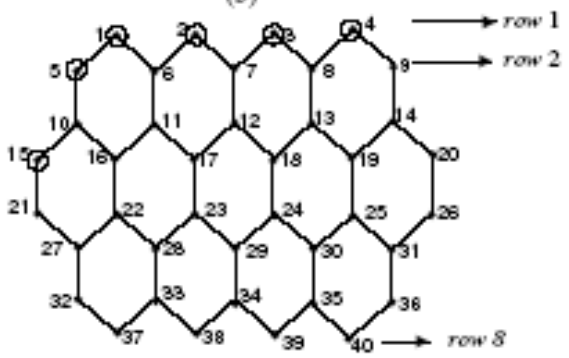

(c)

Figure 3: Circled vertices constitute (a) a minimum zero forcing set of circum-corene(1) (b) a minimum zero forcing set of circum-naphthalenes(1) (c) a minimum zero forcing set of circum-anthracenes (1)

\section{Zero Forcing Algorithm in Circum-Coronene}

Input: Circum-coronene $(n), n \geq 1$.

Algorithm: Name the vertices of Circum-Coronene $(n), n \geq 1$ as 1 to $6 n^{2}$ sequentially from left to right, row wise begining with the first row. Consider $2 n+3$ hexagons in outer most layer of the $\operatorname{Circum-corenes}(n)$. Let $P^{*}$ denote the path induced by the edges of the hexagons that are not boundary edges of any other hexagon. Select $2 n+3$ vertices of degree 2 in $P^{*}$, which are at distance 2 apart on $P^{*}$. Apart from these vertices, select one more vertex in $S_{n}$ from $P^{*}$, select one more vertex in $S_{n}$ from $P^{*}$, adjacent to any one vertex which is selected already. See Figure 3(a). 
Output: $Z($ Circum-trizenes $(\mathrm{n}))=2 n+4$.

Proof of Correctness: Every vertex $v \in S_{n}$, is adjacent to exactly one non-colored vertex. Proceeding inductively, every vertex in the graph colored. Now $\left|S_{n}\right|=2 n+4$. This implies that, $Z($ Circum-coronene $(\mathrm{n}))=$ $2 n+4$. Hence the proof.

Lemma 2.9. Let $G$ be a circum-coronene $(n), n \geq 1$. Then $Z(G)=2 n+4$.

\subsection{Zero Forcing of Circum-Polyacenes}

In this section we compute zero forcing number of various n-Circum-Polyacenes. The polyacenes are a class of organic compounds and polycyclic aromatic hydrocarbons made up of linearly fused benzene rings. The larger representatives have potential interest in optoelectronic applications and are actively researched in chemistry and electrical engineering. Some members of polyacenes are naphthalenes $\left(\mathrm{C}_{10} \mathrm{H}_{8}\right)$, anthracenes $\left(\mathrm{C}_{14} \mathrm{H}_{10}\right)$, tetracenes $\left(\mathrm{C}_{18} \mathrm{H}_{12}\right)$, pentacenes $\left(\mathrm{C}_{22} \mathrm{H}_{14}\right)$, hexacenes $\left(\mathrm{C}_{26} \mathrm{H}_{16}\right)$. Circumscribing polyacenes gives $n$-circum-polyacenes which is obtained by circumscribing a set of linear catacondensed benzenoids. Some members of polyacenes when circumscribed are circum-naphthalenes $(n)$ commonly known as ovalenes and circum-ovalenes, circum-anthracenes $(n)$, circum-tetracenes $(n)$, circumpentacenes $(n)$, circum-hexacenes $(n)$.

Let $H_{n}$ denote a linear chain of $\mathrm{n}$ hexagons. Adding $k$ layers of hexagons to the boundary of $H_{n}$ gives rise to various chemical structures as follows: 

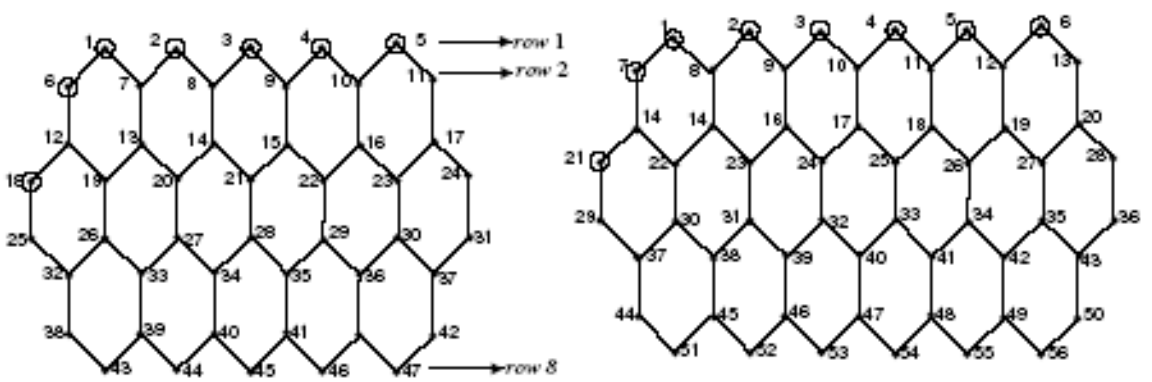

(b)

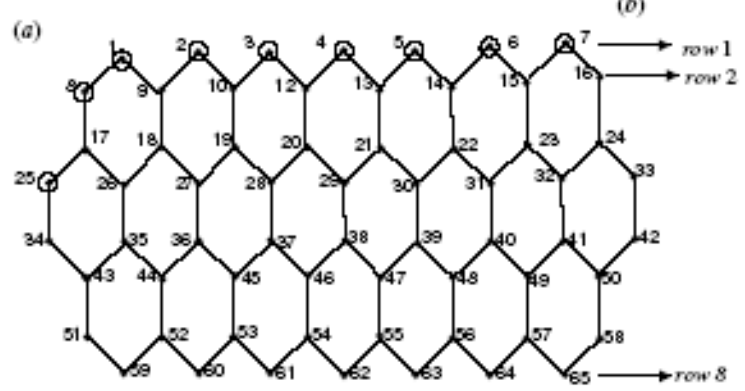

(c)

Figure 4: Circled vertices constitute (a) a minimum zero forcing set of circumtetracenes(1) (b) a minimum zero foreing set of circum-pentacenes(1) (c) a minimum zero forcing set of eireum-hexacenes(1)

1. Circum-naphthalenes (n) when $n=2$ and $k=1, \ldots, n$. See Figure $4(b)$

2. Circum-anthracenes (n) when $n=3$ and $k=1, \ldots, n$. See Figure $4(c)$.

3. Circum-tetracenes (n) when $n=4$ and $k=1, \ldots, n$. See Figure $5(a)$.

4. Circum-pentacenes (n) when $n=5$ and $k=1, \ldots, n$. See Figure $5(b)$.

5. Circum-hexacenes (n) when $n=6$ and $k=1, \ldots, n$. See Figure 6.[7]

Theorem 2.10. Let $G$ be a circum-naphthalenes $(n), n \geq 1$. Then $Z(G)=$ $2 n+3$.

Theorem 2.11. Let $G$ be a circum-anthracenes $(n), n \geq 1$. Then $Z(G)=$ $2 n+4$. 
Theorem 2.12. Let $G$ be a circum-tetracenes $(n), n \geq 1$. Then $Z(G)=$ $2 n+5$.

Theorem 2.13. Let $G$ be a circum-pentacenes $(n), n \geq 1$. Then $Z(G)=$ $2 n+6$.

Theorem 2.14. Let $G$ be a circum-hexacenes $(n), n \geq 1$. Then $Z(G)=$ $2 n+7$.

\section{Conclusion}

In this paper, we have obtained the zero forcing number for pyrene networks, circum-pyrene networks, circum-trizene networks and circum-polyacenes networks.

\section{References}

[1] AIM Minimum Rank - Special Graphs Work Group, "Zero forcing sets and the minimum rank of graphs", Linear algebra and its applications, vol. 428, no. 7, pp. 1628-1648, Apr. 2008, doi: 10.1016/j.laa.2007.10.009.

[2] D. Burgarth, V. Giovannetti, L. Hogben, S. Severini and M. Young, "Logic circuits from zero forcing", Natural computing, vol. 14, no. 3, pp. 485-490, Sep. 2015, doi: 10.1007/s11047-014-9438-5.

[3] M. Gentner, L. Penso, D. Rautenbach and U. Souza, "Extremal values and bounds for the zero forcing number", Discrete applied mathematics, vol. 214, pp. 196-200, Dec. 2016, doi: 10.1016/j.dam.2016.06.004.

[4] T. Haynes, S. Hedetniemi, S. Hedetniemi, and M. Henning, "Power domination in graphs applied to electrical power networks". SIAM journal on discrete mathematics, vol. 15, no. 4, pp. 519-529, 2002, doi: $10.1137 /$ S0895480100375831.

[5] K. Benson, D. Ferrero, M. Flagg, V. Furst, L. Hogben, V. Vasilevska and B. Wissman, "Zero forcing and power domination for product graphs", Feb. 2017. arXiv:1510.02421v4. 
[6] J. Anitha and I. Rajasingh "Power domination parameters in honeycomb-like networks," in Applied mathematics and scientific computing. Trends in mathematics. B. Rushi, R. Sivaraj, B. Prasad, M. Nalliah, and A. Reddy Eds. Cham: Birkhäuser, 2019, pp. 613-621, doi: 10.1007/978-3-030-01123-9_61.

[7] J. Quadras, K. Balasubramanian and K. Arputha Christy, "Analytical expressions for Wiener indices of $\mathrm{n}$-circumscribed pericondensed benzenoid graphs", Journal of mathematical chemistry, vol. 54. no. 3, Mar. 2016, doi: 10.1007/s10910-016-0596-9. 